\title{
Trazodona no Tratamento da Insónia: Qual a Evidência?
}

\section{Trazodone for the Treatment of Insomnia: What is the Evidence?}

Diana S. Mota¹, Maria João Martins

Autor Correspondente/Corresponding Author:

Diana S. Mota [ddianamota@gmail.com] Rua das Casas Novas n 423, 4620-111 Covas Lousada, Portugal ORCID iD: 0000-0001-6080-5538

\section{RESUMO}

INTRODUÇÃO: A insónia constitui uma queixa frequente nos cuidados de saúde primários, com um impacto negativo na qualidade de vida dos utentes. Assim sendo, o seu tratamento adequado é fundamental. A trazodona é um fármaco que tem sido amplamente usado no tratamento da insónia, apesar de estar apenas aprovado, pelo INFARMED e Food and Drug Administration (FDA), para o tratamento da depressão, sendo relevante rever a evidência que suporta este seu uso off-label.

METODOLOGIA: Com recurso a sites de medicina baseada na evidência pesquisaram-se meta-análises, revisões sistemáticas, ensaios clínicos e guidelines, publicadas nas línguas inglesa e portuguesa entre 19/05/2008 e 19/05/2018, usando os termos MeSH "Trazodone" e "Sleep Initiation and Maintenance Disorders" e as palavras-chave "Trazodone" e "Insomnia".

RESULTADOS: Foram incluídos na revisão 10 artigos: 2 meta-análises, 3 revisões sistemáticas, 2 ensaios clínicos e 3 guidelines. As meta-análises revelaram que a trazodona é eficaz no tratamento da insónia (nível de evidência -NE-2), o mesmo se observou nos ensaios clínicos (NE 2) e em duas revisões sistemáticas (NE 2), com a terceira revisão referindo ausência de evidência para retirar conclusões. Relativamente às guidelines, duas suportam o uso da trazodona (Força de Recomendação -FR- B e FR C) enquanto a terceira sugere a sua não utilização (FR B).

CONCLUSÃO: Existe evidência limitada de que a trazodona é eficaz no tratamento da insónia em indivíduos com idade $\geq 18$ anos - força de recomendação B. É, assim, importante, que no futuro, sejam realizados mais estudos para reforçar o uso de trazodona no tratamento da insónia.

PALAVRAS- CHAVE: Distúrbios do Início e da Manutenção do Sono/tratamento; Trazodona/uso terapêutico 


\section{ABSTRACT}

INTRODUCTION: Insomnia is a frequent complaint in primary care, with a negative impact on patients' quality of life. Therefore, its proper treatment is essential. Trazodone is a drug that has been widely used in insomnia treatment, although it is only approved by INFARMED and Food and Drug Administration (FDA) for depression treatment. Therefore, it is relevant to review the evidence supporting this off-label use.

METHODOLOGY: Using evidence-based medicine websites, a research of meta-analyzes, systematic reviews, clinical trials and guidelines were completed by using the MeSH terms "Trazodone" and "Sleep Initiation and Maintenance Disorders" and the keywords "Trazodone" and "Insomnia". Afterwards, the articles published in English and Portuguese between 19/05/2008 and 19/05/2018 were selected.

RESULTS: The review included 10 articles: 2 meta-analyzes, 3 systematic reviews, 2 clinical trials and 3 guidelines. Meta-analyzes revealed that trazodone is effective in treating insomnia (level of evidence-LE-2), as was observed in clinical trials (LE 2) and in two systematic reviews (LE 2), with the third review reporting no evidence to draw conclusions. Regarding the guidelines, two support the use of trazodone (recommendation strength-RS-B and RS C) while the third suggests its non-use (RSB).

CONCLUSION: There is limited evidence that trazodone is effective in insomnia treatment - Recommendation Strength B. It is important that further researches be conducted to reinforce the use of trazodone in insomnia treatment.

KEYWORDS: Sleep Initiation and Maintenance Disorders/drug therapy; Trazodone/therapeutic use

\section{INTRODUÇÃO}

A insónia constitui uma das queixas mais frequentes ao nível dos cuidados de saúde primários. Estudos da sua prevalência indicam que 1/3 dos adultos, em países ocidentais, experiencia dificuldades, pelo menos uma vez por semana, em iniciar ou manter o sono, com cerca de $6 \%$ a 15\% preenchendo os critérios de diagnóstico de insónia. ${ }^{1}$

Esta afeta negativamente a qualidade de vida dos indivíduos, estando associada a um aumento do risco de acidentes e a uma diminuição da concentração, produtividade laboral e desempenho diário em geral. A insónia está também associada a um risco aumentado de patologias mentais (depressão e ansiedade) e cardiovasculares (hipertensão arterial e acidente vascular cerebral).1,

Neste âmbito, é importante que a insónia seja tratada adequadamente para prevenir estas potenciais consequências negativas. Nos Estados Unidos da América, a trazodona constitui um dos fármacos mais prescritos para o tratamento da insónia, apesar de estar apenas aprovada para o tratamento da depressão pela Food and Drug Administration (FDA). Em Portugal, a trazodona também não tem aprovação pelo INFARMED como hipnótico, embora seja amplamente utilizada para esse fim..$^{1-4}$

Assim sendo, revela-se pertinente estudar este uso off-label da trazodona, sendo objetivo deste trabalho rever a evidência da eficácia da trazodona no tratamento da insónia em indivíduos com idade $\geq 18$ anos.

\section{METODOLOGIA}

Em maio de 2018, usando os termos MeSH "Trazodone" e "Sleep Initiation and Maintenance Disorders", foi realizada uma pesquisa de meta-análises, revisões sistemáticas, ensaios clínicos e guidelines, publicadas entre 19/05/2008 e 19/05/2018, nas línguas inglesa e portuguesa, nas seguintes bases de dados: National Guideline Clearing House, National Institute of Health and Care Excellence (NICE), Canadian Medical Association Practice Guidelines, Cochrane, Data base of Abstracts of Reviews of Effectiveness (DARE) e PubMed. Optou-se por associar uma segunda pesquisa usando as palavras-chave "Trazodone" e "Insomnia", uma vez que esta incluía alguns artigos distintos dos identificados anteriormente e com relevância para o tema em revisão. Nesta segunda pesquisa, foram usadas as mesmas bases de dados e limite temporal definidos anteriormente.

Foram excluídos os estudos que não respeitavam o PICO (Tabela 1). Adicionalmente foram excluídos artigos de opinião, artigos de revisão clássica, editoriais, letters e notícias. Foram também excluídos os estudos duplicados ou já incluídos nas revisões sistemáticas ou meta-análises selecionadas.

Para avaliação e atribuição dos níveis de evidência (NE) 
e forças de recomendação (FR) usou-se a escala Strength of Recommendation Taxonomy (SORT) da American Academy of Family Physicians.

\section{RESULTADOS}

Através da pesquisa realizada, obtiveram-se 238 artigos, dos quais 119 foram excluídos por duplicação. Os restantes foram submetidos a leitura do título, resumo ou texto integral, tendo sido excluídos 97 artigos por não estarem de acordo com o PICO (Tabela 1) e/ou não respeitarem a tipologia de artigos definidos. Adicionalmente, foram excluídos 12 artigos por estarem abrangidos nas meta-análises e/ou revisões sistemáticas selecionadas.

\section{TABELA 1. PICO.}

\section{$\mathrm{PICO}$}

\begin{tabular}{|c|c|}
\hline População & $\begin{array}{l}\text { Indivíduos com idade } \geq 18 \text { anos com diagnósti- } \\
\text { co de insónia }\end{array}$ \\
\hline Intervenção & Trazodona \\
\hline Comparativo & $\begin{array}{l}\text { Placebo ou outros tratamentos (fármacos, } \\
\text { terapia cognitivo-comportamental) }\end{array}$ \\
\hline Outcome & $\begin{array}{l}\text { Melhoria subjetiva de um ou mais dos seguintes } \\
\text { parâmetros do sono: qualidade, duração total, } \\
\text { tempo até início do sono ou número de desper- } \\
\text { tares noturnos }\end{array}$ \\
\hline
\end{tabular}

No final deste processo obtiveram-se 10 artigos: duas meta-análises, três revisões sistemáticas, dois ensaios clínicos e três guidelines. O resumo da evidência dos artigos selecionados encontra-se representado nas Tabelas 2 a 5.

\section{META-ANÁLISES}

A meta-análise de Everitt $H$ et al (2018) incluiu três ensaios clínicos aleatorizados ( $\mathrm{n}=370)$ comparando a trazodona (25 mg a 150 mg) com o placebo em indivíduos com insónia primária ou secundária, com follow-up variável entre 7 dias e 6 meses. Os autores concluíram que "existe evidência equívoca suportando o uso de trazodona no tratamento da insónia a curto prazo, não existindo evidência suportando o seu uso a longo prazo". Os ensaios clínicos incluídos nesta meta-análise eram de baixa qualidade, como referido pelos autores, devido à ausência ou limitação de informação sobre a randomização, a allocation concealed (ocultação da sequência de alocação) e o blinding (ocultação dos profissionais, participantes e avaliação dos resultados). Assim sendo, foi atribuído um nível de evidência 2 (Tabela 2). ${ }^{1}$

Por sua vez, a meta-análise de Yi XY et al (2018) englobou sete ensaios clínicos aleatorizados, mas apenas três avaliaram parâmetros subjetivos do sono ( $n$ = 358). Estes também compararam a trazodona (50 a 150 mg) com o placebo em indivíduos com insónia pri-

TABELA 2. Resumo Meta-análises e Nível de Evidência (NE) atribuído.

\begin{tabular}{|c|c|c|c|c|c|}
\hline \multicolumn{6}{|c|}{ META-ANÁLISE } \\
\hline $\begin{array}{l}\text { Referência } \\
\text { Bibliográfica }\end{array}$ & $\begin{array}{l}\text { Estudos } \\
\text { incluídos }\end{array}$ & Intervenção & Resultados & Conclusão & NE \\
\hline $\begin{array}{l}\text { Everitt H } \\
\text { etal } \\
2018\end{array}$ & $\begin{array}{l}3 \text { ECA } \\
\text { - } 2 \text { insónia } \\
\text { primária } \\
\text { - } 1 \text { insónia } \\
\text { secundária } \\
\mathrm{n}=370\end{array}$ & $\begin{array}{l}\text { Trazodona } \\
\text { (25 a } 150 \text { mg) } \\
\text { vs } \\
\text { placebo } \\
\text { Outcome: eficácia, segu- } \\
\text { rança e tolerabilidade } \\
\text { - parâmetros subjetivos }\end{array}$ & $\begin{array}{l}\text { Houve uma ligeira melhoria } \\
\text { subjetiva da qualidade do sono } \\
\text { no grupo tratado com trazodona } \\
\text { comparativamente ao grupo com } \\
\text { placebo (SMD =-0,34; } 95 \% \text { IC } \\
\left.-0,66 \text { a - } 0,02 ;\left.\right|^{2}=49 \%\right)\end{array}$ & $\begin{array}{l}\text { Os estudos incluídos demonstraram uma } \\
\text { evidência equívoca suportando o uso de } \\
\text { trazodona no tratamento da insónia a curto } \\
\text { prazo. Não existe evidência suportando o seu } \\
\text { uso a longo prazo. } \\
\text { Dados insuficientes para retirar conclusões } \\
\text { relativamente à segurança. }\end{array}$ & 2 \\
\hline $\begin{array}{l}\text { YiXY } \\
\text { et al (2018) }\end{array}$ & $\begin{array}{l}7 \text { ECA } \\
-2 \text { insónia } \\
\text { primária } \\
\text { - } 5 \text { insónia } \\
\text { secundária } \\
n=429\end{array}$ & $\begin{array}{l}\text { Trazodona } \\
\text { (50 a } 150 \text { mg) } \\
\text { vs } \\
\text { placebo } \\
\text { Outcome: } \\
\text { eficácia e tolerabilidade } \\
\text { - } 4 \text { ECA: parâmetros } \\
\text { objetivos } \\
\text { - } 3 \text { ECA: parâmetros } \\
\text { subjetivos }\end{array}$ & $\begin{array}{l}\text { Pacientes tratados com trazodo- } \\
\text { na percecionaram uma melhoria } \\
\text { subjetiva da qualidade do sono } \\
\text { (2 medidos pelo "Pittsburgh Sleep } \\
\text { Quality Index" e } 1 \text { pelo "Morning } \\
\text { Questionnaire") comparativamen- } \\
\text { te ao grupo tratado com placebo } \\
\text { (SMD= -0,41; } 95 \% \text { IC -0,82 a - } \\
\text { 0,00, } p=0,05 ; I^{2}=65 \%, p=0,06 \text { ) } \\
\text { Em termos de tolerabilidade, não } \\
\text { houve diferenças significativas } \\
\text { entre o grupo trazodona e o } \\
\text { grupo placebo relativamente a } \\
\text { descontinuação por efeitos ad- } \\
\text { versos. (OR = 0,86; } 95 \% \text { IC 0,28 } \\
\text { a 2,63, } p=0,80 ; I^{2}=0 \% \text {, } \\
\text { p=0,41) }\end{array}$ & $\begin{array}{l}\text { Trazodona é um hipnótico efetivo e bem } \\
\text { tolerado no tratamento da insónia primária e } \\
\text { secundária. } \\
\text { No entanto, a falta de dados claros da sua } \\
\text { eficácia e segurança na insónia, assim com a } \\
\text { ausência de aprovação pela FDA, alertam para } \\
\text { uma precaução na sua utilização. } \\
\text { A falta de dados também não permitiu realizar } \\
\text { recomendações relativamente à duração do } \\
\text { tratamento. } \\
\text { A trazodona apresenta boa tolerância no } \\
\text { tratamento da insónia a curto prazo. }\end{array}$ & 2 \\
\hline
\end{tabular}


TABELA 3. Resumo Revisões Sistemáticas e Nível de Evidência (NE) atribuído.

\begin{tabular}{|c|c|c|c|c|c|}
\hline $\begin{array}{l}\text { Referência } \\
\text { Bibliográfica }\end{array}$ & $\begin{array}{l}\text { Estudos } \\
\text { incluídos }\end{array}$ & Intervenção & Resultados & Conclusão & NE \\
\hline $\begin{array}{l}\text { Jaffer K } \\
\text { etal } \\
2017\end{array}$ & $\begin{array}{l}45 \text { estudos } \\
5 \text { insónia } \\
\text { primária } \\
40 \text { insónia } \\
\text { secundária } \\
n=3780\end{array}$ & $\begin{array}{l}\text { Trazodona } \\
\text { (10 a } 600 \text { mg) } \\
\text { vs } \\
\text { placebo e outros } \\
\text { tratamentos (fármacos } \\
\text { hipnóticos e terapia cog- } \\
\text { nitivo-comportamental) } \\
\text { Outcome: eficácia e } \\
\text { segurança } \\
\text { - } 11 \text { estudos: parâme- } \\
\text { tros objetivos ( } n=198 \text { ) } \\
\text { - } 19 \text { estudos: parâme- } \\
\text { tros subjetivos } \\
\text { ( } n=2871 \text { ) } \\
\text { - } 11 \text { estudos: parâme- } \\
\text { tros objetivos e subjeti- } \\
\text { vos ( } n=470 \text { ) } \\
\text { - } 4 \text { estudos: parâmetros } \\
\text { desconhecidos ( } n=241 \text { ) }\end{array}$ & $\begin{array}{l}\text { A maioria dos estudos (43/45) } \\
\text { concluiu que a trazodona é efetiva } \\
\text { no tratamento da insónia, sendo } \\
\text { que em } 21 \text { dos } 45 \text { estudos a insónia } \\
\text { era secundária à depressão ou à } \\
\text { terapêutica com antidepressivos. } \\
\text { Os estudos demonstram que a } \\
\text { trazodona é efetiva aumentando a } \\
\text { duração total do sono e reduzindo o } \\
\text { "tempo até início do sono". Estudos } \\
\text { com duração do tratamento de 6-12 } \\
\text { semanas demonstraram também } \\
\text { uma melhoria significativa na quali- } \\
\text { dade do sono. }\end{array}$ & $\begin{array}{l}\text { A revisão da literatura sugere que existe } \\
\text { evidência adequada suportando a eficácia } \\
\text { e segurança da trazodona em baixa dose no } \\
\text { tratamento da insónia, particularmente em } \\
\text { pacientes deprimidos. } \\
\text { Apesar dos resultados encorajadores, } \\
\text { estudos de maiores dimensões usando } \\
\text { parâmetros objetivos são necessários para } \\
\text { melhor compreender a eficácia da trazodo- } \\
\text { na no tratamento da insónia. }\end{array}$ & 2 \\
\hline $\begin{array}{l}\text { Thaler K } \\
\text { etal } \\
2012\end{array}$ & $\begin{array}{l}2 \text { ECA } \\
\text { insónia em } \\
\text { pacien- } \\
\text { tes com } \\
\text { depressão } \\
\text { major } \\
n=353\end{array}$ & $\begin{array}{l}\text { Trazodona } \\
\text { (100 a } 400 \mathrm{mg} \text { ) } \\
\text { vs } \\
\text { fluoxetina } \\
\text { ( } 20 \text { a } 60 \mathrm{mg} \text { ) } \\
\text { ou } \\
\text { venlafaxina } \\
\text { (75 a } 200 \mathrm{mg} \text { ) } \\
\text { Outcome: } \\
\text { eficácia } \\
\text { - parâmetros subjetivos } \\
\text { - duração: } 6 \text { semanas }\end{array}$ & $\begin{array}{l}\text { Os resultados da escala "Hamil- } \\
\text { ton Depressions Rating Scale sleep } \\
\text { disturbance factor" foram superiores } \\
\text { no grupo tratado com trazodona } \\
\text { comparativamente ao grupo com } \\
\text { fluoxetina (-2,7 vs -1,6; } p=0,001) \text {. } \\
\text { Os resultados da escala "Hamil- } \\
\text { ton Depressions Rating Scale sleep } \\
\text { disturbance factor" foram superiores } \\
\text { no grupo tratado com trazodona } \\
\text { comparativamente ao grupo com } \\
\text { venlafaxina ( } 1,42 \text { vs } 2,22 ; p<0,5)\end{array}$ & $\begin{array}{l}\text { A evidência atual não é adequada para } \\
\text { retirar conclusões acerca da superioridade } \\
\text { de qualquer um dos fármacos no tratamen- } \\
\text { to da insónia em pacientes com depressão } \\
\text { major. }\end{array}$ & 2 \\
\hline $\begin{array}{l}\text { Kolla B } \\
\text { et al } \\
2011\end{array}$ & $\begin{array}{l}3 E C \\
\text { insónia em } \\
\text { pacientes } \\
\text { em desin- } \\
\text { toxicação } \\
\text { alcoólica } \\
n=244\end{array}$ & $\begin{array}{l}\text { Trazodona } \\
\text { ( } 50 \text { a } 300 \mathrm{mg} \text { ) } \\
\text { vs } \\
\text { placebo } \\
\text { vs } \\
\text { gabapentina } \\
\text { (300 a } 1800 \mathrm{mg} \text { ) } \\
\text { Outcome: } \\
\text { eficácia } \\
\text { - parâmetros subjetivos } \\
\text { - parâmetros objetivos }\end{array}$ & $\begin{array}{l}\text { Um ensaio clínico controlado } \\
\text { (trazodona (50-200 mg) vs placebo; } \\
\text { n=16) demonstrou uma melhoria } \\
\text { significativa dos resultados nas } \\
\text { escalas "Clinical Global Impression" e } \\
\text { "Hamilton Depressions Rating Scale" } \\
\text { no grupo tratado com trazodona. } \\
\text { Um ensaio clínico controlado } \\
\text { (trazodona (50-150 mg) vs placebo; } \\
\text { n=173) demonstrou uma melhoria } \\
\text { significativa na qualidade do sono } \\
\text { através da escala "Pittsburgh Sleep } \\
\text { Quality Index". } \\
\text { Um ensaio clínico controlado } \\
\text { (gabapentina (300-1800 mg) vs } \\
\text { trazodona (50-300 mg); n = 55) de- } \\
\text { monstrou uma melhoria no sono em } \\
\text { ambos grupos, medido pela "Sleep } \\
\text { Problems Questionnaire". No entanto, } \\
\text { essa melhoria foi superior no grupo } \\
\text { tratado com gabapentina. }\end{array}$ & $\begin{array}{l}\text { A trazodona é o fármaco com mais evidên- } \\
\text { cia de eficácia no tratamento da insónia em } \\
\text { pacientes em desintoxicação alcoólica, mas } \\
\text { essa evidência é ambígua. }\end{array}$ & 2 \\
\hline
\end{tabular}

$E C=$ ensaio clínico; $E C A=$ ensaio clínico aleatorizado

mária ou secundária com um período de follow-up de 1 a 4 semanas. Conclui-se que "a trazodona é um hipnótico efetivo e bem tolerado no tratamento da insónia primária e secundária. No entanto, a falta de dados claros da sua eficácia e segurança, assim com a ausência de aprovação pela FDA, alertam para uma precaução na sua uti- lização. A falta de dados também não permitiu realizar recomendações relativamente à duração do tratamento." Tal como na meta-análise anterior, os ensaios clínicos incluídos apresentam baixa qualidade, devido, dependendo do estudo, ao tamanho da amostra, follow-up inadequado (< 80\%) e à ausência ou limitações na descri- 
TABELA 4. Resumo Ensaios Clínicos e Nível de Evidência (NE) atribuído.

\begin{tabular}{|c|c|c|c|c|c|}
\hline \multicolumn{6}{|c|}{ ENSAIOS CLÍNICOS } \\
\hline $\begin{array}{l}\text { Referência } \\
\text { Bibliográfica }\end{array}$ & $\begin{array}{l}\text { Tipo de estudo } \\
\text { e amostra }\end{array}$ & Intervenção & Resultados & Conclusão & NE \\
\hline $\begin{array}{l}\text { Ilinoiu GA } \\
\text { et al } \\
2015\end{array}$ & $\begin{array}{l}\text { ECA } \\
\text { Insónia residual em } \\
\text { pacientes com depres- } \\
\text { são major tratados } \\
\text { com inibidores seleti- } \\
\text { vos da recaptação da } \\
\text { serotonina (SSRIs) } \\
\text { (Fluvoxamina 75-150 } \\
\text { mg/dia, Sertralina } \\
\text { 50-100 mg/dia, fluo- } \\
\text { xetina 20-40 mg/dia) } \\
\text { n= } 32 \\
\text { - Trazodona: } 12 \\
\text { - Pregabalina: } 10 \\
\text { - Mirtazapina: } 10\end{array}$ & $\begin{array}{l}\text { Trazodona + SSRIs } \\
\text { ( } 50 \text { a } 150 \mathrm{mg} \text { ) } \\
\text { vs } \\
\text { Mirtazapina + SSRIs } \\
\text { (15 a } 30 \mathrm{mg} \text { ) } \\
\text { vs } \\
\text { Pregabalina + SSRIs } \\
\text { (75 a } 150 \text { mg) } \\
\text { Outcome: } \\
\text { Eficácia } \\
\text { e tolerabilidade } \\
\text { - parâmetros } \\
\text { subjetivos } \\
\text { Duração: } \\
24 \text { semanas }\end{array}$ & $\begin{array}{l}\text { Às } 24 \text { semanas, os doentes dos três } \\
\text { grupos reportaram melhoria na } \\
\text { qualidade do sono no "Pittsburgh } \\
\text { Sleep Quality Index", com uma ten- } \\
\text { dência de superioridade no grupo } \\
\text { da trazodona (-38,7\% vs - } 31,2 \% \\
\text { pregabalina vs 30,2\% mirtazapina). }\end{array}$ & $\begin{array}{l}\text { Associação de trazodona, pre- } \\
\text { gabalina ou mirtazapina com } \\
\text { inibidores seletivos da recap- } \\
\text { tação da serotonina provaram } \\
\text { ser úteis em pacientes idosos } \\
\text { com depressão major e insónia } \\
\text { residual, com uma efetividade } \\
\text { ligeiramente superior para a } \\
\text { trazodona e uma melhor } \\
\text { tolerabilidade da mirtazapina. }\end{array}$ & 2 \\
\hline $\begin{array}{l}\text { Paterson } \\
\text { et al } \\
2009\end{array}$ & $\begin{array}{l}\text { ECA } \\
\text { Insónia primária } \\
\mathrm{n}=12\end{array}$ & $\begin{array}{l}\text { Trazodona } \\
\text { (100 mg) } \\
\text { vs } \\
\text { placebo } \\
\text { Outcome: } \\
\text { Eficácia } \\
\text { - parâmetros subjetivos } \\
\text { - parâmetros objetivos } \\
\text { Duração: } \\
\text { 1 semana }\end{array}$ & $\begin{array}{l}\text { Houve uma melhoria significativa } \\
\text { nos parâmetros subjetivos do sono: } \\
\text { o "adormecer" e "qualidade do } \\
\text { sono" melhoraram no "Leeds Sleep } \\
\text { Evaluation Questionnaire", sem afe- } \\
\text { tar negativamente o "despertar" } \\
\text { e o "comportamento após acordar", } \\
\text { indicando ausência de efeito signifi- } \\
\text { cativo de ressaca. } \\
\text { De forma similar, foi observado me- } \\
\text { Ihoria no score de "satisfação com o } \\
\text { sono" e "qualidade do sono" no "St. } \\
\text { Mary's Hospital Sleep Questionnaire". }\end{array}$ & $\begin{array}{l}\text { Os dados sugerem que } \\
\text { administração aguda de } \\
\text { trazodona à noite é um promo- } \\
\text { tor efetivo do sono na insónia } \\
\text { primária e pode ser útil no tra- } \\
\text { tamento da insónia intermédia } \\
\text { e continuidade do sono. }\end{array}$ & 2 \\
\hline
\end{tabular}

ECA = ensaio clínico aleatorizado; SSRIs = Inibidores seletivos da recaptação da serotonina

TABELA 5. Resumo Guidelines e Força de Recomendação (FR) atribuída.

\begin{tabular}{|c|c|c|}
\hline \multicolumn{3}{|l|}{ GUIDELINES } \\
\hline $\begin{array}{l}\text { Referência } \\
\text { Bibliográfica }\end{array}$ & Recomendação & FR \\
\hline $\begin{array}{l}\text { American } \\
\text { Academy of Sleep } \\
\text { Medicine }\end{array}$ & \multirow[t]{2}{*}{$\begin{array}{l}\text { Sugerem não usar a trazodona como tratamento } \\
\text { para a insónia inicial ou intermédia em adultos. }\end{array}$} & \multirow[t]{2}{*}{ B } \\
\hline $\begin{array}{l}\text { Sateia Metal } \\
2017\end{array}$ & & \\
\hline $\begin{array}{l}\text { Brazilian Sleep } \\
\text { Association }\end{array}$ & \multirow{2}{*}{$\begin{array}{l}\text { Relativamente ao tratamento farmacológico o } \\
\text { zolpidem foi indicado como o fármaco padrão } \\
\text { no tratamento da insónia, sendo recomendado } \\
\text { como segunda linha o zopiclone, trazodona e } \\
\text { doxepina. } \\
\text { A dose de trazodona recomendada foi } 50 \mathrm{mg} / \text { dia. }\end{array}$} & \multirow[t]{2}{*}{ B } \\
\hline $\begin{array}{l}\text { Pinto Let al } \\
2010\end{array}$ & & \\
\hline $\begin{array}{l}\text { British Association } \\
\text { for Psychophar- } \\
\text { macology }\end{array}$ & \multirow{2}{*}{$\begin{array}{l}\text { A evidência da eficácia da doxepina, trimipra- } \\
\text { mina, trazodona e paroxetina no tratamento da } \\
\text { insónia é limitada. } \\
\text { Considerar antidepressivos quando existe } \\
\text { concomitantemente uma perturbação do humor, } \\
\text { mas neste caso usar a dose terapêutica da per- } \\
\text { turbação do humor. }\end{array}$} & \multirow[t]{2}{*}{ C } \\
\hline $\begin{array}{l}\text { Wilson SJ et al } \\
2010\end{array}$ & & \\
\hline
\end{tabular}

ção da randomização, allocation concealed e blinding. Foi, assim, atribuído a esta meta-análise um Nível de Evidência 2 (Tabela 2). ${ }^{2}$

\section{REVISÕES SISTEMÁTICAS}

A revisão sistemática de Jaffer $K$ et al (2017) avaliou 45 estudos $(n=3780)$ comparando a trazodona (10 a 600 mg) com o placebo e outros tratamentos (fármacos hipnóticos e terapia cognitivo-comportamental), maioritariamente em indivíduos com insónia secundária (40 estudos), com follow-up variável entre 1 dia e 6 meses. Destes, 30 estudos avaliaram parâmetros subjetivos do sono ( $n=3341)$. Esta revisão sugere que "existe evidência adequada suportando a eficácia e segurança da trazodona em baixa dose no tratamento da insónia, particularmente em pacientes deprimidos." Apesar dos resultados encorajadores, os autores referem que são necessários mais estudos para melhor compreender a eficácia da trazodona no tratamento da insónia. Os estudos incluídos nesta revisão sistemática apresentam limitações na sua qualidade, relacionados com o tamanho da amostra, tempo de follow-up e processo ou descrição da randomização, allocation concealed e blinding, 
tendo, por isso, sido atribuído um nível de evidência 2 (Tabela 3). ${ }^{3}$

Por sua vez, a revisão sistemática de Thaler Ket al (2012) incluiu dois ensaios clínicos aleatorizados ( $n=353$ ) comparando o uso de trazodona (100 a 400 mg) vs fluoxetina (20 a 60 mg) ou venlafaxina (75 a 200 mg) no tratamento da insónia em pacientes com depressão major, após um período de follow-up de 6 semanas. Os autores concluíram que "a evidência atual não é adequada para retirar conclusões acerca da superioridade de qualquer um dos fármacos." A esta revisão foi atribuído um nível de evidência 2, tendo em conta a baixa qualidade dos ensaios clínicos incluídos, relacionada com limitações na descrição da metodologia de randomização, allocation concealed e blinding (Tabela 3). ${ }^{5}$

Relativamente à revisão sistemática de Kolla B et al (2011), englobou três ensaios clínicos ( $n=244$ ) avaliando o uso de trazodona (50 a 300 mg) no tratamento da insónia em pacientes em desintoxicação alcoólica, com um follow-up variável de 1 a 3 meses. A trazodona foi comparada com o placebo em dois estudos e com a gabapentina (300 a 1800 mg) em um estudo. Conclui-se que a "trazodona é o fármaco com mais evidência de eficácia no tratamento da insónia em pacientes em desintoxicação alcoólica, mas essa evidência é ambígua". Mais uma vez, a qualidade dos estudos incluídos é baixa, sendo os motivos similares aos referidos anteriormente, pelo que também se atribuiu a esta revisão sistemática um nível de evidência 2 (Tabela 3). ${ }^{6}$

\section{ENSAIOS CLÍNICOS}

O ensaio clínico de Ilinoium GA et al (2015) avaliou o uso de trazodona (50 a 150 mg) vs mirtazapina (15 a 30 mg) ou pregabalina (75 a 150 mg) no tratamento da insónia residual em pacientes com depressão major tratados com inibidores seletivos da recaptação da serotonina (ISRS) ( $n$ = 32). $O$ estudo teve uma duração de 24 semanas e demonstrou que a "associação de trazodona, pregabalina ou mirtazapina com ISRS são úteis em pacientes idosos com depressão major e insónia residual, com uma efetividade ligeiramente superior da trazodona e uma melhor tolerabilidade da mirtazapina." Dado o tamanho amostral, limitações na descrição do processo de aleatorização e não referência ao processo de blinding, foi atribuído a este estudo um nível de evidência 2 (Tabela 4). ${ }^{7}$

Por sua vez, o ensaio clínico de Paterson LM et al (2009) comparou o uso de trazodona (100 mg) vs placebo em indivíduos com insónia primária ( $\mathrm{n}=12$ ) após uma 1 semana de follow-up. Os autores concluíram que "a administração aguda de trazodona à noite é um promotor efetivo do sono na insónia primária". Foi também atribuí- do a este estudo um nível de evidência 2, dado o tamanho amostral e limitações na descrição do processo de aleatorização, blinding e follow-up (Tabela 4). ${ }^{8}$

\section{GUIDELINES}

A guideline da American Academy of Sleep Medicine (2017) foi o único artigo dos selecionados que não recomendou o uso de trazodona no tratamento da insónia em adultos. Trata-se de uma guideline baseada na evidência, cuja recomendação se fundamentou num único ensaio clínico comparando a trazodona (50 mg) com o placebo e o zolpidem (10 mg) ( $n=187$ ) e cujos resultados não demonstraram uma melhoria clinicamente significativa nos parâmetros do sono. Os autores atribuíram a esse ensaio uma qualidade moderada, definindo a recomendação de "não uso da trazodona" como fraca, segundo a escala GRADE. Assim sendo, foi atribuído a esta guideline uma força de recomendação B (Tabela 5). ${ }^{\text {? }}$

Por sua vez, a guideline da Brazilian Sleep Association (2010) recomenda o uso de trazodona como tratamento de segunda linha na insónia em adultos. Trata-se também de uma guideline baseada na evidência, cuja recomendação foi classificada como nível 3 e 4, segundo a escala de qualidade dessa associação. Através da análise dessa escala e dos estudos incluídos, foi atribuído uma força de recomendação B. Este é o único artigo que faz recomendações relativamente à dose de trazodona a usar - 50 mg (Tabela 5). ${ }^{10}$

Por fim, a guideline da British Association for Psychopharmacology (2010) refere que a "evidência da eficácia da trazodona no tratamento da insónia é limitada", recomendando que os antidepressivos sejam apenas considerados quando existe concomitantemente uma perturbação do humor, usando a dose terapêutica aprovada para essa perturbação do humor. Esta guideline baseia-se na opinião de peritos, pelo que Ihe foi atribuído uma Força de Recomendação C (Tabela 5). ${ }^{11}$

\section{DISCUSSÃO E CONCLUSÃO}

As duas meta-análises selecionadas demonstraram que a trazodona é eficaz no tratamento da insónia (NE 2). mesmo se observou em duas revisões sistemáticas (NE 2), com a terceira referindo ausência de evidência para concluir sobre a superioridade da trazodona relativamente à fluoxetina e venlafaxina (NE 2), não sendo, contudo, possível excluir a sua eficácia vs placebo, uma vez que esta não foi avaliada. Por sua vez, os dois ensaios clínicos também demonstraram a eficácia da trazodona (NE 2). Relativamente às guidelines, duas suportam o uso da trazodona (FR B e FR C) enquanto uma sugere a sua não utilização (FR B). 
Tendo em conta o referido anteriormente, podemos concluir que existe evidência limitada de que a trazodona é eficaz no tratamento da insónia em indivíduos com idade $\geq 18$ anos - força de recomendação $B$.

Embora relevante para a prática clínica diária, a evidência obtida nesta revisão não é suficiente para realizar recomendações relativamente à dose e duração do tratamento, dado a grande variabilidade entre os diferentes estudos. Apesar disso, estes parecem sugerir uma eficácia da trazodona na insónia em doses inferiores às usadas no tratamento da depressão, com a maioria avaliando uma duração de tratamento inferior a 3 meses.

Outras das limitações desta revisão é a variabilidade das escalas utlizadas quer no diagnóstico da insónia quer na avaliação dos resultados (outcome) do tratamento com trazodona. Adicionalmente, alguns dos estudos incluídos avaliavam nos seus resultados (outcome) tanto parâmetros objetivos como subjetivos do sono, não sendo possível excluir a potencial contribuição dos parâmetros objetivos para a conclusão dos estudos analisados e da própria revisão, embora as autoras estivessem atentas a esse aspeto e valorizassem apenas os resultados subjetivos.

É, assim, importante que sejam realizados mais estudos nesta temática, nomeadamente de qualidade superior, com inclusão de maior número de indivíduos, uniformização dos resultados (outcomes) e avaliação da eficácia e segurança da trazodona a longo prazo.

CONFLITOS DE INTERESSE: Os autores declaram não ter qualquer conflito de interesse na realização do presente trabalho.

FONTES DE FINANCIAMENTO: Não houve qualquer fonte de financiamento na realização do presente trabalho.

PROVENIÊNCIA E REVISÃO POR PARES: Não comissionado; revisão externa por pares.

CONFLICTS OF INTEREST: The authors declare that they have no conflicts of interest.

FINANCIAL SUPPORT: This work has not received any contribution, grant or scholarship.

PROVENANCE AND PEER REVIEW: Not commissioned; externally peer reviewed.

\section{REFERÊNCIAS}

1. Everitt $H$, Baldwin S, Stuart B, Lipinska G, Mayers A, Malizia $\mathrm{AL}$, et al. Antidepressants for insomnia in adults. Cochrane Database Syst Rev. 2018; 5. doi:10.1002/14651858.CD010753. pub2.

2. Yi XY, Ni SF, Ghadami MR, Meng HQ. Chen MY, Juang L, et al. Trazodone for the treatment of insomnia: a meta-analysis of randomized placebo-controlled trials. Sleep Med. 2018;45:2532. doi:10.1016/j.sleep.2018.01.010.

3. Jaffer KY, Chang T, Vanle B, Dang J, Steiner A, Loera $\mathrm{N}$ et al. Trazodone for Insomnia: A Systematic Review. Innov Clin Neurosci. 2017;14(7-8):24-34.

4. INFARMED. Prontuário terapêutico. [consultado em 20/05/2018]. Disponível em https://app10.infarmed.pt/ prontuario/framepesactivos.php?palavra=TRAZODO$N A \& x=0 \& y=0 \& r b 1=0$,

5. Thaler KJ, Morgan L, Van Noord M, Gaynes B, Hansen R, Lux $L$, et al. Comparative effectiveness of second-generation antidepressants for accompanying anxiety, insomnia, and pain in depressed patients: a systematic review. Depress Anxiety. 2012;29:495-505. doi: 10.1002/da.21951.

6. Kolla BP, Mansukhani M, Schneekloth T. Pharmacological treatment of insomnia in alcohol recovery: a systematic review. Alcohol Alcohol. 2011;46:578-85. doi: 10.1093/alcalc/ agr073.

7. Ilinoiu GA, Bratu R, Androne F. Psychopharmacological strategies for residual insomnia in old age depression treated with selective serotonin reuptake inhibitors. Eur Neuropsychopharmacol. 2015; 25: S92-3.

8. Paterson LM, Nutt D, Durant C, Wilson S. Efficacy of Trazodone in primary insomnia: A double-blind randomised placebo controlled polysomnographic study. Eur Neuropsychopharmacol. 2009; 19: S385-6. doi: 10.1016/S0924-977X(09)70593-0.

9. Sateia M, Buysse D, Krystal A, Neubauer D, Heald J. Clinical practice guideline for the pharmacologic treatment of chronic insomnia in adults: an American Academy of Sleep Medicine clinical practice guideline. J Clin Sleep Med. 2017;13:307-49. doi: $10.5664 / j c s m .6470$.

10. Pinto L, Alves R, Caixeta E, Fontenelle J, Bacellar A, Poyares D, et al. New guidelines for diagnosis and treatment of insomnia. Arq Neuropsiquiatr. 2010;68:666-75.

11. Wilson S, Nutt D, Alford C, Argyropoulos S, Baldwin D, Bateson A, et al. British Association for Psychopharmacology consensus statement on evidence-based treatment of insomnia, parasomnias and circadian rhythm disorders. J Psychopharmacol. 2010; 24:1577-601. doi: 10.1177/0269881110379307. 